\title{
Late-life depression in Peru, Mexico and Venezuela: the 10/66 population-based study
}

Mariella Guerra, Cleusa P. Ferri, Ana Luisa Sosa, Aquiles Salas, Ciro Gaona,

Victor Gonzales, Gabriela Rojas de la Torre and Martin Prince

\section{Background}

The proportion of the global population aged 60 and over is increasing, more so in Latin America than any other region. Depression is common among elderly people and an important cause of disability worldwide.

\section{Aims}

To estimate the prevalence and correlates of late-life depression, associated disability and access to treatment in five locations in Latin America.

\section{Method}

A one-phase cross-sectional survey of 5886 people aged 65 and over from urban and rural locations in Peru and Mexico and an urban site in Venezuela. Depression was identified according to DSM-IV and ICD-10 criteria, Geriatric Mental State-Automated Geriatric Examination for Computer Assisted Taxonomy (GMS-AGECAT) algorithm and EURO-D cut-off point. Poisson regression was used to estimate the independent associations of sociodemographic

characteristics, economic circumstances and health status with ICD-10 depression.

\section{Results}

For DSM-IV major depression overall prevalence varied between $1.3 \%$ and $2.8 \%$ by site, for ICD-10 depressive episode between $4.5 \%$ and $5.1 \%$, for GMS-AGECAT depression between $30.0 \%$ and $35.9 \%$ and for EURO-D depression between $26.1 \%$ and $31.2 \%$; therefore, there was a considerable prevalence of clinically significant depression beyond that identified by ICD-10 and DSM-IV diagnostic criteria. Most older people with depression had never received treatment. Limiting physical impairments and a past history of depression were the two most consistent correlates of the ICD-10 depressive episode.

\section{Conclusions}

The treatment gap poses a significant challenge for Latin American health systems, with their relatively weak primary care services and reliance on private specialists; local treatment trials could establish the cost-effectiveness of mental health investment in the government sector.

\section{Declaration of interest}

The 10/66 Dementia Research Group works closely with Alzheimer's Disease International, the non-profit federation of 77 Alzheimer associations around the world. Alzheimer's Disease International is supported in part by grants from GlaxoSmithKline, Novartis, Lundbeck, Pfizer and Eisai.
The prevalence of late-life depression has been extensively studied in high-income countries in the European, North American and Asian Pacific regions. ${ }^{1,2}$ The main influence on prevalence seems to be the criterion used to make the diagnosis. In late life, depressive disorder diagnosed according to rigorous research criteria such as DSM-IV ${ }^{3}$ major depression and ICD $-10^{4}$ depressive episode is uncommon, with a weighted mean prevalence of only $1.8 \%$; when all those with clinically relevant symptoms are included, the weighted mean prevalence rises to $13.3 \% .^{1}$ The relative lack of epidemiological data from low- and middleincome countries is particularly striking - in the two systematic reviews, all of the 34 publications covering the period 19891996 and the 122 papers covering the period 1993-2004 described research carried out in high-income countries. Three more recent publications report an unusually high prevalence of late-life depression according to clinical diagnostic criteria. The prevalence of DSM-IV major depression in Nigeria was $7.1 \%{ }^{5}$ and that of ICD-10 depressive episode in Brazil was $19.2 \% .^{6}$ In both settings, depression was associated with high levels of social disability and impaired quality of life. In a study from Peru published as an institutional report, the prevalence of DSM-IV major depression was $9.8 \%{ }^{7}$ More research is needed to determine whether these findings are typical of lower-income regions in general. In the multicentre SABE (Salud, Bienstar y Envejecimiento en America Latina y el Caribe) study the prevalence of clinically significant depression (a score of 11 or more on the Geriatric Depression Scale) in the six Latin American capital cities ranged from $16.5 \%$ to $30.1 \%$ in women and from $11.8 \%$ to $19.6 \%$ in men. ${ }^{8}$
This is broadly consistent with prevalences reported in two cross-national comparisons of late-life depression in Europe: the ten country SHARE (Survey of Health, Ageing and Retirement in Europe) study ${ }^{9}$ in which a validated cut-off point was applied to the EURO-D depression scale ${ }^{10}$, and the EURODEP study ${ }^{11}$ in which depression was diagnosed using the Geriatric Mental StateAutomated Geriatric Examination for Computer Assisted Taxonomy (GMS-AGECAT). ${ }^{12,13}$

Using a common methodology across all locations, we set out to estimate the prevalence of late-life depression in three urban and two rural locations in three Latin American countries (Mexico, Peru and Venezuela) according to four different diagnostic criteria, (DSM-IV major depression, ICD-10 depressive episode, GMSAGECAT and EURO-D depression). We also aimed to assess levels of disability associated with ICD-10 depressive episode and subsyndromal depression, and to describe access to treatment. Finally we aimed to estimate the independent associations of sociodemographic characteristics, economic circumstances and health status with ICD-10 depressive episode in each site.

\section{Method}

\section{Setting and study design}

The full 10/66 study protocol has been published elsewhere. ${ }^{14} \mathrm{~A}$ one-phase cross-sectional population-based survey has been conducted of all those over 65 years old from defined catchments areas for each of the five locations. For urban catchment areas, predominantly middle-class or professional areas with high- 
income earners were avoided. Rural catchment areas were defined by low population density and traditional agrarian lifestyle. The total target sample was 2000 for each country. In Peru and Mexico, we sampled from urban and rural districts, whereas in Venezuela we sampled from an urban district only. In Peru the urban catchment areas comprised two districts (Lima Cercado and San Miguel) in the capital city, Lima. The rural catchment areas included six districts (Cerro Azul, Imperial, Nuevo Imperial, Quilmana, San Luis and San Vicente) in the coastal province of Canete. The main economic activities are fishing and agriculture. In Mexico the urban catchment areas were six districts in the suburb of Tlalpan in the south of Mexico City. The rural catchment areas included nine villages in the north of the state of Morelos, a mountainous district $70 \mathrm{~km}$ from Mexico City. The main economic activity is agriculture. In Venezuela, we sampled an urban district, Caricuao in the south west of the capital city, Caracas, where most participants came from low- or middle-income backgrounds and lived in social housing apartments.

\section{Measurements}

\section{Depression}

The diagnosis of depression was determined according to four different criteria, all derived from the same comprehensive structured clinical interview, the Geriatric Mental State (GMS). ${ }^{12}$ The prevalence of each condition was determined with respect to the past month.

Depression of clinical significance. The Geriatric Mental State is supported by AGECAT, ${ }^{13}$ a diagnostic algorithm based on clinical principles. For the community B3 version of the GMS this generates four syndrome clusters: organicity (dementia); schizophrenia and related paranoia; depression; and anxiety neurosis. A diagnostic confidence level is provided for each syndrome, ranging from 0 (no symptoms) to 5 (very severely affected). Level three and greater represent a degree of severity warranting professional intervention and levels one and two subcases. These stage one diagnoses are then organised into a single stage two diagnosis on the basis of precedence determined by a hierarchically structured algorithm. In the $10 / 66$ pilot study ${ }^{15}$ sensitivity was consistently higher for the stage one than for the stage two depression diagnosis, against the Montgomery-Åsberg Depression Scale rating. This is explained by the tendency of the AGECAT hierarchical system to overdiagnose organicity in lower-income settings, and then to accord this precedence over depression in the hierarchical determination. For this reason, we used the stage one GMS-AGECAT depression syndrome for this analysis - this is subsequently referred to as 'GMS-AGECAT depression'.

The EURO- $\mathrm{D}^{10}$ is a symptom scale originally developed to compare symptoms of late-life depression across 11 European countries in the EURODEP consortium. It is derived from the GMS and covers 12 symptom domains: depressed mood, pessimism, suicidality, guilt, sleep, interest, irritability, appetite, fatigue, concentration, enjoyment and tearfulness. Each item is scored 0 (symptom not present) or 1 (symptom present), and item scores are summed to produce a scale with a minimum score of 0 and a maximum of 12. Its cross-cultural measurement properties have been extensively investigated, in the European EURODEP and SHARE studies, as well as in the 10/66 pilot studies in Latin America, India and China. ${ }^{15-17}$ Although the scale has moderately high internal consistency (standardized alpha value varied from 0.58 to 0.80$),{ }^{17}$ two underlying factors are reliably identified (affective suffering and motivation). In the EURODEP study the optimum cut-off point was $\geqslant 4$ for the identification of DSM-IV major depression and GMS-AGECAT depression, ${ }^{17}$ and this cut-off point was applied in the subsequent SHARE study of the prevalence of late-life depression across Europe. Those scoring four or more on the scale are subsequently described as cases of 'EURO-D depression'. We used the term 'subsyndromal depression' to describe cases of EURO-D depression, with symptoms that did not meet criteria for ICD-10 depressive episode.

Diagnostic criteria for depression. Diagnoses of DSM-IV major depression and ICD-10 depressive episode were both derived using a computerised algorithm applied to GMS. The ICD-10 divides depressive episodes into mild, moderate and severe. The DSM-IV, but not ICD-10, specifies that symptoms should be severe enough to cause 'clinically significant distress or impairment' and excludes a diagnosis of major depression if the symptoms are better accounted for by bereavement.

\section{Other health conditions}

Other recorded health conditions were: dementia according to the $10 / 66$ dementia diagnosis algorithm; ${ }^{18}$ self-reported stroke; and having 3 or more of 11 limiting physical impairments (arthritis or rheumatism; eyesight problems; hearing difficulty or deafness; persistent cough; breathlessness, difficulty breathing or asthma; high blood pressure; heart trouble or angina; stomach or intestine problems; faints or blackouts; paralysis, weakness or loss of one leg or arm; skin disorders such as pressure sores, leg ulcers or severe burns).

\section{Disability}

We used the World Health Organization Disability Assessment Schedule II (WHODAS-II) ${ }^{19}$ to measure limitation and participation restriction. It was developed by the WHO as a culture-fair assessment tool for use in cross-cultural comparative epidemiological and health services research.

\section{Sociodemographic status}

Participants' ages were established from participant and informant report and an official identification document; when the information was not clear or there was a discrepancy, an event calendar was used. Information was also collected on marital status, education (none, did not complete primary, completed primary, secondary, tertiary) and social support (we used 'never or less than monthly contacts' with family and friends as a proxy measure of lack of social support).

\section{Socioeconomic circumstances}

This was assessed using different measures. It was determined whether participants received any income, any pension, proxy measure of household income (sum of total income for households with more than one participant); a household assets index was calculated (number of assets in the household categorised: $0-2 ; 3-5$ and 6 or more assets); and food insecurity was assessed by the question 'Do you ever go hungry because there is not enough food to eat?'

\section{Ethics}

Participants were recruited following informed signed consent. People with dementia who lack capacity for consent were recruited on the basis of a relative's signed agreement. Illiterate persons were read the information sheet and consent form, and invited to 
express their consent verbally, which was witnessed. Studies were approved by local ethical committees as well as by the ethical committee of King's College London (College Research Ethics Committee).

\section{Statistical analysis}

We recorded the sociodemographic characteristics, economic circumstances and health status of the sample in each site. We identified the prevalence of depression in each setting for each of the four outcomes: EURO-D depression, GMS-AGECAT depression, ICD-10 depressive episode and DSM-IV major depression, by age and gender, with $95 \%$ confidence intervals adjusted for household clustering. We determined levels of disability (mean WHODAS-II global disability scores and the proportion reporting 15 or more disability days in the past month) according to depression status, dividing each sample into those with no depression (neither ICD-10 depressive episode nor EURO-D depression), subsyndromal depression (EURO-D depression not confirmed as depressive episode by ICD-10), and ICD-10 depressive episode. We then estimated the effects of subsyndromal depression and ICD-10 depressive episode on the proportion reporting 15 or more disability days (severe disability) using Poisson regression, adjusting for age, gender, 10/66 dementia and number of limiting physical impairments. The population attributable prevalence fraction for subsyndromal depression and ICD-10 depressive episode was then estimated using the STATA for Windows 10.1, which aflogit command estimates the attributable fraction from within the Poisson regression framework, thus enabling confounders to be taken into account. Population attributable prevalence fractions when calculated from prevalence ratios in cross-sectional studies represent the proportion of prevalent severe disability that could theoretically be avoided if the exposure could be removed from the population, taking into account the effect of the exposure on both incidence and duration of the severe disability state, assuming a causal relationship estimated free of confounding.

Finally, we used a Poisson regression model (adjusted for household clustering) to estimate the independent associations of the following factors with ICD-10 depressive episode:

(a) sociodemographic characteristics - age, gender, marital status and level of education social support;

(b) never or less than monthly contact with family and with friends;

(c) economic circumstances - household income and food insecurity; the effect of other correlated indicators of socioeconomic position (receiving any income, yes/no; receiving any pension, yes/no; and numbers of household assets) was tested by substituting household income with each of them in turn;

(d) health status - 10/66 dementia, number of physical impairments, past depression and stroke.

\section{Results}

\section{General characteristics}

The numbers of participants interviewed in each site was 1933 for Peru (urban, 1381; rural, 552); 2002 for Mexico (urban, 1002; rural, 1000) and 1951 for Venezuela (urban only). Response rates were $80 \%$ and above in all locations $(80 \%, 88 \%, 84 \%, 86 \%$ and $80 \%$ respectively).

Online Table DS1 describes the general characteristics of the samples by country. Age distributions were similar for each site, other than Venezuela where there was a relative preponderance of younger participants. Nearly two-thirds of participants were female in each site, other than in rural Peru (53.4\%). Levels of education were lower in rural than in urban locations, especially rural Mexico where $83.7 \%$ of the participants had minimal or no education. Participants in rural locations also reported fewer household assets and a higher prevalence of food insecurity. Whereas two-thirds or more of participants across all locations reported receiving some kind of income, pension coverage was especially low in rural Mexico (25.4\%). For a high proportion of the participants social contact was limited to family members; only a small proportion (from $5.4 \%$ in urban Peru to $8.6 \%$ in rural Peru) had less than monthly or no contact with family members, but a much higher proportion had less than monthly or no contact with friends $(13.1 \%$ in rural Peru to $59.8 \%$ in rural Mexico). Half or more of the participants reported at least one limiting physical impairment and nearly one in five reported three or more. A past history of depression was especially common in urban Peru $(34.9 \%)$, in other locations the prevalence varied between $13.6 \%$ and $18.1 \%$. The prevalence of self-reported stroke was lower in rural Peru (3.6\%) than other locations $(5.7 \%$ to $8.2 \%$ ). Dementia prevalence varied from $6.7 \%$ in rural Peru to $11.1 \%$ in rural Mexico.

\section{Prevalence of depression}

The prevalence of depression was consistently much higher for EURO-D and GMS-AGECAT depression than for DSM-IV major depression and ICD-10 depressive episode (Table DS2). For EURO-D depression overall prevalence varied between 26.1\% and $31.2 \%$ by location, for GMS-AGECAT depression between $30.0 \%$ and $35.9 \%$, for DSM-IV major depression between $1.3 \%$ and $2.8 \%$ and for ICD-10 depressive episode between $4.5 \%$ and $5.1 \%$. There was therefore relatively little variation in the prevalence of depression between locations, according to any of the criteria. Prevalence according to DSM-IV and ICD-10 diagnostic criteria was a little lower in rural compared with urban locations, but the same trend was not seen for EURO-D or GMS-AGECAT depression. In all locations, for all or most criteria the prevalence of depression was higher in women than among men, and varied somewhat with age, but with no consistent trend. Given the much higher prevalence of EURO-D depression compared with that of ICD-10 depressive episode, we explored the phenomenology of these two categories, empirically, by comparing the frequency of individual EURO-D symptoms and mean EURO-D total scores according to depression status. The mean total EURO-D scores were 1.0 (s.d.=1.1) for those with neither condition, 5.3 (s.d.=1.4) for those with subsyndromal depression (EURO-D depression cases not meeting ICD-10 criteria) and 7.1 $($ s.d. $=1.8)$ for those with ICD-10 depressive episode. Symptom prevalences were similar between subsyndromal depression and ICD-10 depressive episode cases for most EURO-D symptoms (depression, pessimism, wishing death, guilt, sleep disturbance, irritability and tearfulness). However, loss of interest (56\% v. $22 \%)$, loss of appetite ( $49 \%$ v. 30\%), fatigue ( $88 \% v .56 \%)$, loss of concentration $(57 \%$ v. 35\%) and loss of enjoyment $(54 \% v$. $18 \%)$ were all considerably more common among those meeting ICD-10 criteria (Table DS3).

\section{Impact of depression}

For those with subsyndromal depression, levels of disability were intermediate between those with no depression and those with ICD-10 depressive episode, according to both mean WHODASII global disability scores and the proportion with severe disability as indicated by having reported 15 or more disability days in the 
past month. After adjusting for age, gender, number of limiting physical impairments and dementia both subsyndromal depression and ICD-10 depressive episode were independently associated with the prevalence of severe disability, in each country. Those with subsyndromal depression were one and a half to two times more likely to report severe disability. The population attributable prevalence fractions suggest that $13.6 \%$ of the prevalence of severe disability in Peru, $32.4 \%$ in Mexico and $31.7 \%$ in Venezuela could be independently attributed to depression. In each country, given the higher prevalence of subsyndromal compared with ICD-10 depression, a similar or greater proportion of severe disability prevalence was attributed to subsyndromal depression compared with ICD-10 depressive episode (Table 1).

\section{Treatment of depression}

Most of those with an ICD-10 depressive episode did not report having ever received in-patient or out-patient treatment for depression: $75.9 \%$ in urban Peru, $81.2 \%$ in rural Peru, $87.2 \%$ in urban Mexico, $95.6 \%$ in rural Mexico and $80.4 \%$ in Venezuela. The proportions that had visited any healthcare provider in the past 3 months were $69.0 \%, 62.5 \%, 80.9 \%, 66.7 \%$ and $75.7 \%$ respectively.

\section{Factors associated with depression}

Table 2 presents the crude and adjusted prevalence ratios for the association of sociodemographic characteristics, economic circumstances and health status with ICD-10 depressive episode, in each location. After adjusting for other covariates, there was a trend towards a lower prevalence of depression with increasing age in Venezuela only. Univariate associations between female gender and depression in all three urban locations were no longer statistically significant after adjustment; closer inspection of the models suggested that the main confounders were living without a partner, limited social support, physical impairment and, for urban locations only, level of education. There was a trend towards a protective effect of higher levels of education in all locations other than rural Mexico; however, this effect was statistically significant only in urban Peru. Of the indicators of socioeconomic status, food insecurity was strongly positively associated with depression in Mexico, with non-significant trends in Peru and Venezuela. Receiving any income was inversely associated with depression in rural Mexico only. Neither receiving a pension, nor number of household assets was associated with depression. Limited contact with family was associated with depression in urban Mexico only. For limited contact with friends there was a trend towards a univariate association with depression in all locations, but after adjustment the effect was statistically significant only in rural Mexico. Increasing numbers of physical impairments and a past history of depression were consistently associated with an increased prevalence of depression in all locations.

\section{Discussion}

In our study of late-life depression in three Latin American countries, the prevalence depends mainly upon the diagnostic criterion, with little variation evident between locations. The most restrictive criterion, DSM-IV major depression, returns the lowest prevalence with ICD-10 depressive episode only slightly more inclusive. The DSM-IV, but not ICD-10, specifies that symptoms should be severe enough to cause 'clinically significant distress or impairment' and excludes a diagnosis of major depression if the symptoms are better accounted for by bereavement. The prevalence of EURO-D and GMS-AGECAT depression is around 6 times higher than that of ICD-10 depressive episode and 15 times higher than that of DSM-IV major depression. These findings are all consistent with earlier reviews based largely on European and North American data. ${ }^{15}$ The prevalence of the broader EURO-D and GMS-AGECAT depression is also consistent with that recorded for the Geriatric Depression Scale in the Latin American SABE study ${ }^{8}$ and with the relatively high prevalence of EURO-D depression recorded in the three 'Latin' countries (France, Spain and Italy) in the European SHARE study.

Given the large discrepancy between the prevalence of late-life depression according to ICD-10 and DSM-IV clinical criteria, and the broader EURO-D and GMS-AGECAT depression syndromes, it was important to establish whether the subsyndromal cases detected by EURO-D but not meeting ICD-10 criteria were indeed 'clinically significant'. The strong and independent association between subsyndromal depression and an established criterion of relatively severe disability, 15 or more disability days in the past month, would appear to indicate that the DSM-IV and ICD-10 diagnostic criteria might indeed be missing a substantial proportion of clinically significant cases. We have also demonstrated, as others have shown with respect to younger adults, ${ }^{20,21}$ that the true population burden of depression is significantly underestimated by these very narrow diagnostic definitions.

\begin{tabular}{|c|c|c|c|c|c|}
\hline & \multirow[b]{2}{*}{$n(\%)$} & \multirow[b]{2}{*}{$\begin{array}{l}\text { WHODAS-Il score } \\
\text { Mean (s.d.) }\end{array}$} & \multicolumn{3}{|c|}{ Severe disability (15 or more disability days in the past month) } \\
\hline & & & Proportion (\%) & $\begin{array}{l}\text { Adjusted prevalence } \\
\text { ratio }(95 \% \mathrm{Cl})\end{array}$ & $\begin{array}{c}\text { Population attributable } \\
\text { prevalence fraction }(95 \% \mathrm{Cl})\end{array}$ \\
\hline Peru & & $\mathrm{MD}=12$ & $\mathrm{MD}=444$ & $n=1444$ & \\
\hline Not depressed & $1346(71.5)$ & $8.0(14.2)$ & 226/1048 (21.6) & 1 (ref) & - \\
\hline EURO-D depression only & $434(23.0)$ & $16.1(17.9)$ & $114 / 326 \quad(35.0)$ & $1.56(1.29-1.89)$ & $10.6(7.3-13.7)$ \\
\hline ICD-10 depressive episode & $103 \quad(5.5)$ & $27.8(22.4)$ & $26 / 71 \quad(36.6)$ & $1.53(1.09-2.14)$ & $3.0(1.3-4.7)$ \\
\hline Mexico & & $\mathrm{MD}=3$ & $\mathrm{MD}=22$ & $n=1961$ & \\
\hline Not depressed & 1409 (71.1) & $7.0(13.8)$ & $87 / 1393(6.2)$ & 1 (ref) & - \\
\hline EURO-D depression only & $482(24.3)$ & $15.1(20.6)$ & $77 / 479(16.1)$ & $2.15(1.57-2.94)$ & $18.5(11.4-25.1)$ \\
\hline ICD-10 depressive episode & $92(4.6)$ & $28.3(23.4)$ & 28/90 (31.1) & $3.41(2.31-5.02)$ & $13.9(10.0-17.7)$ \\
\hline Venezuela & & $M D=119$ & $\mathrm{MD}=625$ & $n=1330$ & \\
\hline Not depressed & $1357(70.3)$ & $6.6(11.4)$ & 49/893 (5.5) & 1 (ref) & - \\
\hline EURO-D depression only & $466(24.1)$ & $16.9(17.7)$ & 43/358 (12.0) & $2.01(1.33-3.03)$ & 15.5 (6.7-23.5) \\
\hline ICD-10 depressive episode & 107 (5.5) & $34.1(26.1)$ & $24 / 87 \quad(27.6)$ & $4.34(2.48-7.60)$ & $16.2(10.6-21.4)$ \\
\hline
\end{tabular}




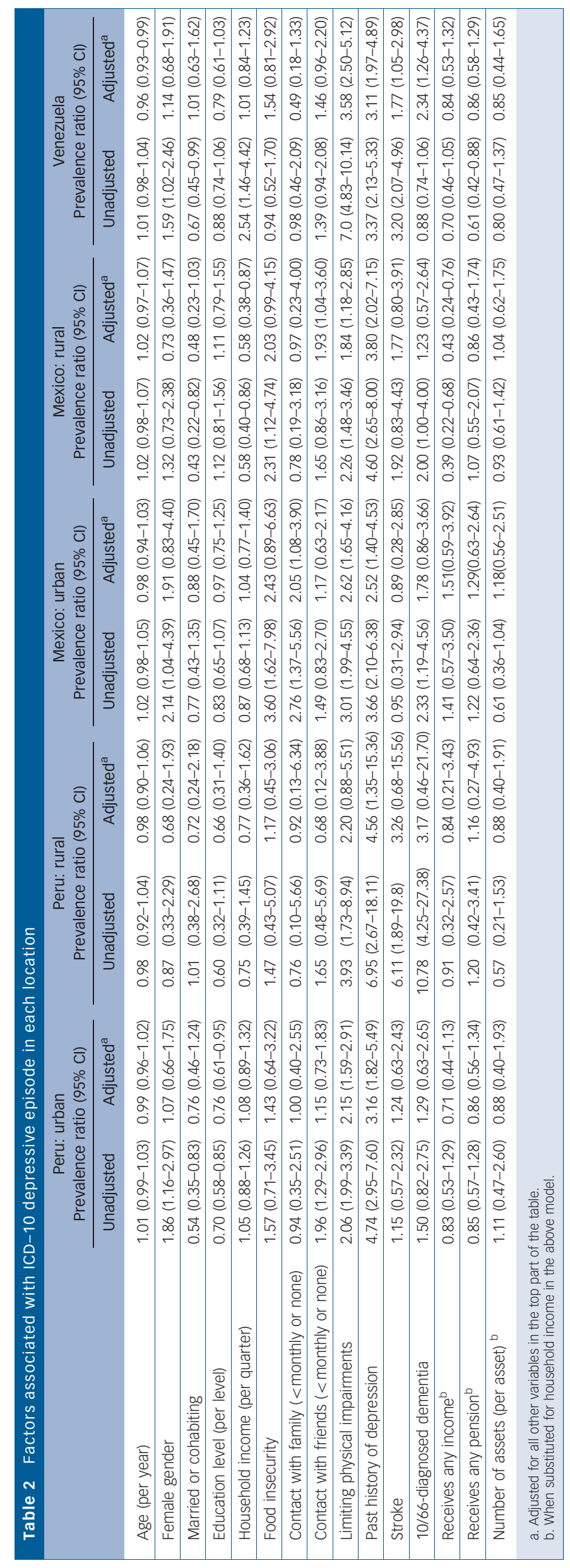

In the World Mental Health Surveys there was a considerable treatment gap for all non-psychotic mental disorders, greater for high-income than for low- and middle-income countries. ${ }^{22}$ For depression among adults, $29.3 \%$ of individuals in high-income countries and $8.1 \%$ of individuals in low- and middle-income countries were receiving treatment. Unfortunately, we only collected data on lifetime treatment, but still found that the large majority of people with ICD-10 depressive episode $(75.9 \%$ to $95.6 \%$ by location) had never received treatment. The proportion receiving treatment for the current episode would obviously be significantly lower. Despite this, the majority of those with ICD-10 depressive episode had consulted a healthcare provider in the past 3 months, signifying that in many cases the opportunity for depression to be diagnosed and treated was there, but had been missed.

To date there have been no prospective studies of potential aetiological factors for late-life depression in low- and middleincome countries. There have, however, been a large number of well-designed cohort studies carried out in Europe and North America, the findings from which have been subject to systematic review $^{2,23}$ and quantitative meta-analysis. ${ }^{23}$ There is strong and fairly consistent evidence to support an increased risk for incident depression associated with female gender, disability, prior depression, bereavement and sleep disturbance.

We are limited in the inferences that we can make from our findings because of the cross-sectional design; associations may have been inflated because of information bias and we cannot determine direction of causality. Nevertheless our findings are broadly consistent with those from incidence studies in highincome countries; limiting physical impairments and a past history of depression were the two factors most consistently correlated with the prevalence of ICD-10 depressive episode.

The finding of a strong and consistent cross-sectional association between depression and physical impairment is, in any case, of practical significance. A recent comprehensive review of interactions between physical and mental health provided strong evidence that, for many chronic physical diseases, comorbidity with depression complicates help-seeking, diagnosis and treatment (particularly through reduced adherence), and hence influences the prognosis of the physical health condition. ${ }^{24}$

The strength of the association with past history of depression is of obvious concern given the extent of lifetime under-identification and treatment apparent in all of the locations. The prevalence of depression was higher among women in all settings in nearly all age groups and for all diagnostic criteria, but this effect was confounded; women were less likely than men to be living with a partner, and were more likely to have a past history of depression, and to have limiting physical impairments. A similar pattern was reported in the SABE study. ${ }^{8}$

There have been many reports from cross-sectional community surveys, from a variety of cultures, of associations between late-life depression and disadvantage with respect to educational level, occupational social class and income. ${ }^{2}$ These are highly correlated variables, and it is difficult to determine the effect of one independent of the others. The possibility of reverse causality also needs to be considered - those whose adult life has been scarred by depression may experience lifelong occupational and economic disadvantage. In our study, there was very little evidence for an association between socioeconomic position and late-life depression prevalence. Level of education seemed to be more relevant than income or wealth. However, food insecurity was strongly positively associated with depression in Mexico, with non-significant trends in Peru and Venezuela.

Some of the heterogeneity in associations across locations in the current analysis may be explained by limited statistical power; 
a clearer picture of the pattern of correlations with late-life depression will be obtained when findings on associations across the full set of 10/66 research centres in Latin America, India, China and Africa are subjected to quantitative meta-analysis. More robust information regarding aetiology will become available when the incidence phase of the project, now underway in most locations, is completed.

\section{Implications}

In summary, our cross-sectional surveys suggest that the prevalence of late-life depression in Peru, Mexico and Venezuela is similar to that seen in Europe. There exists a considerable prevalence of clinically significant depression beyond that identified by the current ICD-10 and DSM-IV diagnostic criteria. Both ICD-10 depressive episode and subsyndromal depression are independently associated with disability, and a similar or even greater proportion of severe disability at population level may arise from subsyndromal cases. The large majority of older people with depression, even those meeting the more exacting DSM-IV and ICD-10 criteria, have never received treatment. Evidence suggests that there are effective treatments available for late-life depression, ${ }^{25}$ although no trials have been carried out on older people in Latin America or other low- and middle-income countries settings. The extent of the current treatment gap poses a significant challenge for Latin American healthcare systems, with their relatively weak primary care services and a traditional reliance on private specialists - local treatment trials may help to establish the cost-effectiveness of investment and training in the government sector.

Mariella Guerra, MD, Universidad Peruana Cayetano Heredia, Institute de la Memoria y Desordenes Relacionadas, Lima, Peru and Centre for Public Mental Health, Health Service and Population Research Department, Institute of Psychiatry, King's Health Service and Population Research Department, Institute of Psychiatry, King's Service and Population Research Department, Institute of Psychiatry, King's College London, UK; Ana Luisa Sosa, MD, The Cognition and Behavior Unit, National Institute of Neurology and Neurosurgery of Mexico, Autonomous National University of Mexico, Mexico City, Mexico; Aquiles Salas, MD, Medicine Department, Caracas University Hospital, Faculty of Medicine, Universidad Central de Venezunela, Caracas, Vencla ciro MD, Institute de la Memoria y Desordenes Relacionadas, Lima, Peru; Gabriela Rojas MD, Institute de la Memoria y Desordenes Relacionadas, Lima, Peru; Gabriela Rojas de la Torre, BSc, The Cognition and Behavior Unit, National Institute of Neurology and Neurosurgery of Mexico, Autonomous National University of Mexico, Mexico City, Mexico; Martin Prince, MD, Centre for Public Mental Health, Health Service and Population Research Department, Institute of Psychiatry, King's College London, UK

Correspondence: Mariella Guerra, Alzheimer's Association, Avda. Araquipa 3845, Miraflores, Lima 18, Peru. Email: mariella.guerra@kcl.ac.uk

First received 2 Feb 2009, final revision 21 May 2009, accepted 17 Jun 2009

\section{Funding}

This study was funded by the US Alzheimer Association (IIRG-04-1286-Mexico and Peru) and by the Fondo Nacional de Ciencia y Tecnologia, Consejo de Desarrollo Cientifico y Humanistico, and Universidad Central de Venezuela (Venezuela).

\section{References}

1 Beekman AT, Copeland JR, Prince MJ. Review of community prevalence of depression in later life. Br J Psychiatry 1999; 174: 307-11.

2 Djernes JK. Prevalence and predictors of depression in populations of elderly: a review. Acta Psychiatr Scand 2006; 113: 372-87.

3 American Psychiatric Association. Diagnostic and Statistical Manual of Mental Disorder (4th edn) (DSM-IV). APA, 1994.

4 World Health Organization. The ICD-10 Classification of Mental and Behavioral Disorders. Diagnostic Criteria for Research. WHO, 1992.
5 Gureje O, Kola L, Afolabi E. Epidemiology of major depressive disorder in elderly Nigerians in the Ibadan Study of Ageing: a community-based survey. Lancet 2007; 370: 957-64.

6 Costa E, Barreto SM, Uchoa E, Firmo JO, Lima-Costa MF, Prince M. Prevalence of International Classification of Diseases, 10th Revision common mental disorders in the elderly in a Brazilian community: The Bambui Health Ageing Study. Am J Geriatr Psychiatry 2007; 15: 17-27.

7 Instituto Especializado de Salud Mental 'Honorio Delgado - Hideyo Nogushi'. Estudio Epidemiologico Metropolitano en Salud Mental [Metropolitan Epidemiological Study of Mental Health in Lima, Peru], vol 18 (1-2): 142. Instituto Especializado de Salud Mental 'Honorio Delgado - Hideyo Nogushi', 2002.

8 Alvarado BE, Zunzunegui MV, Beland F, Sicotte M, Tellechea L. Social and gender inequalities in depressive symptoms among urban older adults of Latin America and the Caribbean. J Gerontol B Psychol Sci Soc Sci 2007; 62: S226-36.

9 Castro-Costa E, Dewey M, Stewart R, Banerjee S, Huppert F, Mendonca-Lima C, et al. Prevalence of depressive symptoms and syndromes in later life in ten European countries: the SHARE study. Br J Psychiatry 2007; 191: 393-401.

10 Prince MJ, Reischies F, Beekman AT, Fuhrer R, Jonker C, Kivela SL, et al. Development of the EURO-D scale - a European Union initiative to compare symptoms of depression in 14 European centres. Br J Psychiatry 1999; 174: 330-8.

11 Copeland JR, Beekman AT, Braam AW, Dewey ME, Delespaul P, Fuhrer R, et al. Depression among older people in Europe: the EURODEP studies. World Psychiatry 2004; 3: 45-9.

12 Copeland JR, Prince M, Wilson KC, Dewey ME, Payne J, Gurland B. The Geriatric Mental State Examination in the 21st century. Int J Geriatr Psychiatry 2002; 17: 729-32.

13 Copeland JR, Dewey ME, Griffiths-Jones HM. A computerized psychiatric diagnostic system and case nomenclature for elderly subjects: GMS and AGECAT. Psychol Med 1986; 16: 89-99.

14 Prince $M$, Ferri $C P$, Acosta $D$, Albanese $E$, Arizaga $R$, Dewey $M$, et al. The protocols for the 10/66 Dementia Research Group population-based research programme. BMC Public Health 2007; 7: 165.

15 Prince M, Acosta D, Chiu H, Copeland J, Dewey M, Scazufca M, et al. Effects of education and culture on the validity of the Geriatric Mental State and its AGECAT algorithm. Br J Psychiatry 2004; 185: 429-36.

16 Castro-Costa E, Dewey M, Stewart R, Banerjee S, Huppert F, Mendonca-Lima $C$, et al. Ascertaining late-life depressive symptoms in Europe: an evaluation of the survey version of the EURO-D scale in 10 nations. The SHARE project. Int J Methods Psychiatr Res 2008; 17: 12-29.

17 Prince MJ, Beekman AT, Deeg DJ, Fuhrer R, Kivela SL, Lawlor BA, et al. Depression symptoms in late life assessed using the EURO-D scale. Effect of age, gender and marital status in 14 European centres. Br J Psychiatry 1999; 174: $339-45$.

18 Prince $M$, Acosta $D$, Chiu $H$, Scazufca $M$, Varghese $M$. Dementia diagnosis in developing countries: a cross-cultural validation study. Lancet 2003; 361: 909-17.

19 Rehm J, Üstün TB, Saxena S, Nelson CB, Chatterji S, Ivis F, et al. On the development and psychometric testing of the WHO screening instrument to assess disablement in the general population. Int J Methods Psychiatr Res 1999; 8: 110-22.

20 Das-Munshi J, Goldberg D, Bebbington PE, Bhugra DK, Brugha TS, Dewey ME, et al. Public health significance of mixed anxiety and depression: beyond current classification. Br J Psychiatry 2008; 192: 171-7.

21 Wells KB, Stewart A, Hays RD, Burnam MA, Rogers W, Daniels M, et al. The functioning and well-being of depressed patients. Results from the Medical Outcomes Study. JAMA 1989; 262: 914-9.

22 Ormel J, Petukhova M, Chatterji S, Aguilar-Gaxiola S, Alonso J, Angermeyer $\mathrm{MC}$, et al. Disability and treatment of specific mental and physical disorders across the world. Br J Psychiatry 2008; 192: 368-75.

23 Cole MG, Dendukuri N. Risk factors for depression among elderly community subjects: a systematic review and meta-analysis. Am J Psychiatry 2003; 160: 1147-56.

24 Prince M, Patel V, Saxena S, Maj M, Maselko J, Phillips MR, et al. No health without mental health. Lancet 2007; 370: 859-77.

25 Hunkeler EM, Katon W, Tang L, Williams Jr JW, Kroenke K, Lin EH, et al. Long term outcomes from the IMPACT randomised trial for depressed elderly patients in primary care. BMJ 2006; 332: 259-63. 